\title{
A Brief Review on Image Restoration Techniques
}

\author{
Shilpa Rani \\ Mtech Student \\ CSE Department \\ SBSSTC,Punjab
}

\author{
Sonika Jindal \\ Assistant Professor \\ CSE Department \\ SBSSTC,Punjab
}

\author{
Bhavneet Kaur \\ Assistant Professor \\ CSE Department \\ SBSSTC,Punjab
}

\begin{abstract}
Image restoration is process of recovering the original image by removing noise and blur from image. Image blur is difficult to avoid in many situations like photography, to remove motion blur caused by camera shake, radar imaging to remove the effect of image system response, etc. Image noise is unwanted signal which comes in image from sensor such as thermal or electrical signal and Environmental condition such as rain, snow etc. Researchers have proposed many methods in this regard and in this paper we will examine and discuss different noise and blur models and restoration methods.
\end{abstract}

\section{Keywords}

Degradation model, image noise and blur model, restoration techniques, point spread function (PSF), peak signal to noise Ratio (PSNR).

\section{INTRODUCTION}

Digital images are electronic snapshots of a scene, which composed of typically picture elements in a grid formation known as pixels. Each pixel holds a value which is quantized that represents the tone at a specific point. Images are obtained in areas ranging from everyday photography to astronomy, remote sensing, microscopy, medical imaging etc.

Image restoration uses a priori knowledge of the degradation. It formulates and evaluates the objective criteria of goodness. The distortion in image can be modeled as noise or blur or a degradation function. Unfortunately all images are more or less blurry. This is due to the reason that there is a lot of interference in the camera as well as in the environment. Blurring of an image can be caused by many factors such as movement during the capture process, using wide angle lens, using long exposure times, etc.[1-2].

\subsection{Degradation Model}

The degradation process can be viewed with the following system. The degraded function is low pass filter.

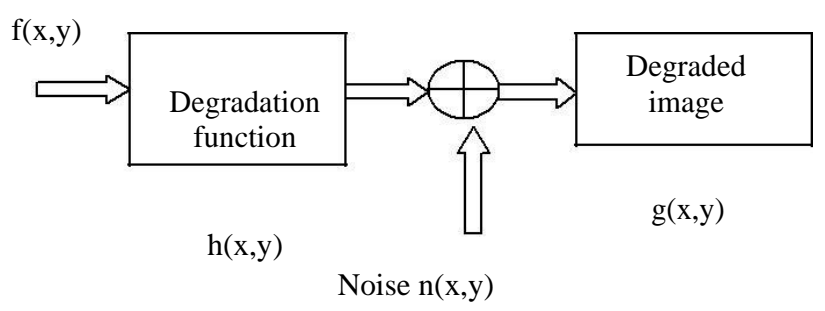

Fig 1: Degeadation Model

The original input is a two-dimensional image $f(x, y)$. This image is operated on the system $\mathrm{h}(\mathrm{x}, \mathrm{y})$ and after the addition of noise $\mathrm{n}(\mathrm{x}, \mathrm{y})$. One can obtain the degraded image $\mathrm{g}(\mathrm{x}, \mathrm{y})$. Digital image restoration may be visualized as a process in which we try to obtain an approximation to $\mathrm{f}(\mathrm{x}, \mathrm{y})$. The blurred image can be described with the following equation. [3] $g(x, y)=h(x, y) * f(x, y)+n(x, y)$

\subsection{Blur model}

\subsubsection{Gaussian blur}

It is type of image blurring filter which use Gaussian function for calculating transformation applied on each pixel. The equation of Gaussian function is

$$
G(x)=\frac{1}{\sqrt{2 \pi \sigma}} e^{\frac{(-x)^{2}}{2 \sigma^{2}}}(2)
$$

Where $\mathrm{x}$ is distance from origin in horizontal axis and $\sigma$ is standard deviation of Gaussian distribution.

\subsubsection{Motion Blur}

Motion blur occur in image due to camera misfocus and change in angle during taking of picture.

\subsubsection{Rectangular blur}

This is blurring in image with specific rectangular area. Blur in image can be identified at any part based on this it can be circular and rectangular.

\subsubsection{Defocus blur}

Defocus blur occurs in image when camera is improperly focused on image. The resolution of image medium depends on amount of defocus. If there is more tolerance of image there is low resolution in image. For good resolution of image defocus in image should be minimize.

\subsection{Noise model}

Noise is unwanted variation in image. It results in change in visibility of an image. Digital images which are related to digital signals are normally corrupted by many types of noise, from which some most occurring noise are Gaussian noise, Salt and pepper noise (Impulse noise), Uniform noise, Brownian Noise, Inverse $\mathrm{f}$ Noise. These noise are represented by following formulae.

\subsubsection{Gaussian noise $P D F$

$$
\text { Gaussian }=\frac{1}{\sqrt{2 \pi \sigma}} e^{\frac{(g-u)^{2}}{2 \sigma^{2}}}
$$

Where

$$
\text { g=gray level }
$$

$\mu=$ mean

$\sigma=$ standard deviation

$$
\begin{aligned}
& \text { 1.3.2 Salt and pepper noise (Impulse noise) } \\
& P D F_{\text {salt and pepper }}=\left\{\begin{array}{c}
A \text { for } g=a(" \text { peeper" } \\
B \text { for } g=b(\text { "salt") }
\end{array}\right.
\end{aligned}
$$

$$
\begin{aligned}
& \text { 1.3.3 Uniform noise } \\
& \qquad P(z)=\left\{\begin{array} { c c } 
{ \frac { 1 } { b - a } } & { \text { if } a \leq z \leq b } \\
{ 0 } & { \text { otherwise } }
\end{array} \left(\begin{array}{l}
(5)
\end{array}\right.\right.
\end{aligned}
$$




$$
\begin{aligned}
& \text { 1.3.4 Brownian Noise } \\
& \qquad W(t)=\int_{0}^{t} d w(t)
\end{aligned}
$$

\section{RESTORATION TECHNIQUES}

\subsection{Median filter}

As the name implies the median filter is statistics method. In this method we find the median of the pixel the replace the pixel by median of the gray levels in their neighborhood of that pixels [2].The median filter is used to remove the salt and pepper noise. It has capability with considerably less blurring than liner smoothing filters of the similar size. In other words we can say that Median filtering is a widely used and very important techniques of filtering and best known for its excellent noise reduction ability from the images.

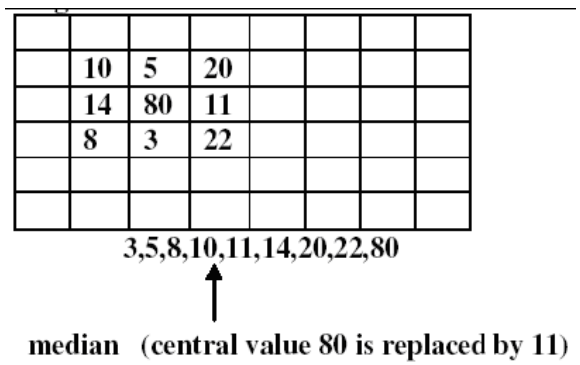

Fig 2: Median filter

By the filtering it keeps the edges while removing the noise. This makes the image not to blur as other smoothing methods. [14]

\subsection{Adaptive filter}

Adaptive filter is type of linear filter that has transfer function controlled by variable parameter. Adaptive filter use the color and gray space for removal of impulsive noise in images. All processing is done on the basis of color and gray space. Adaptive filter are used to remove the effect of speckle noise. This can provide the best noise suppression results and better preserve edges, thin lines and image details and yield better image quality in comparison to other filters.

\subsection{Linear filter}

In linear filter each pixel is replace with linear combination of its neighbors. Image processing operations are implemented with linear filter include sharpening, smoothing and edge enhancement. In linear filter output change linear with input. With the help of linear filter we can easily remove the noise from the image. This filter can be implemented on salt and pepper noise and Gaussian noise. [14]

\subsection{IBD (Iterative Blind Deconvolution)}

Iterative Blind Deconvolution (IBD) was put forward by Ayers and Dainty (1988). It is one of the methods used in blind Deconvolution. This method is based on Fourier Transformation causes less computation. Iterative Blind Deconvolution has good anti-noise capability. In this method image restoration is difficult process where image recovery is performed with little or no prior knowledge of the degrading PSF. The Iterative Blind Deconvolution algorithm has higher resolution and better quality. The main drawback is that convergence of the iterative process is not guaranteed. But the original image can have effect on the final result.

\subsection{NAS-RIF (Nonnegative and Support Constraints Recursive Inverse Filtering)}

The aim of blind Deconvolution is to reconstruct a reliable estimated image from a blurred image. D.Kundur put forward NAS-RIF algorithm (Nonnegative and Support Constraints Recursive Inverse Filtering) to achieve this aim.

In NAS-RIF algorithm based on given image make an estimation of target image. The estimation is made by minimizing an error function which contains the domain of image and nonnegative information of pixels of image. There is a feasible solution that makes the error function globally optimized. In theory, the estimation is equivalent to the real image. The advantage of this algorithm is we don't need to know about the parameters of PSF and the priori information of original image, all we have to determine support domain of target area and to make sure the estimation of image is nonnegative. Another advantage is that this algorithm contains a process which makes sure the function can convergent to global least. The disadvantage of NAS-RIF is that it is sensitive to noise, so it is only proper for images with symmetrical background.

\subsection{Super-resolution restoration algorithm Based on gradient adaptive interpolation}

The basic idea of the gradient-based adaptive interpolation is that the interpolated pixel value is affected by the local gradient of a pixel, mainly in edge areas of the image. The more influence it should have on the interpolated pixel the smaller the local gradient of a pixel. The method involves three subtasks: registration, fusion and deblurring. Firstly we utilize the frequency domain registration algorithm to estimate the motions of the low resolution images. According to the motion the low resolution images are mapped to the uniform high resolution grid, and then the gradient based adaptive interpolation is used to form a high resolution image. Finally, wiener filter is applied to reduce the effects of blurring and noise caused by the system. The main advantage of this algorithm is low computation complexity.[16]

\subsection{Deconvolution Using a Sparse Prior}

This algorithm [7] formulates the Deconvolution problem as given the observation determining the maximum a-posterior estimate of the original image. Furthermore, the algorithm exploits a prior enforcing spatial-domain sparsely of the image derivatives. The resulting non-convex optimization problem is solved using an iterative re-weighted least square method. Although this algorithm has not been natively devised for Poisoning observations, it has been rather successfully applied to raw images. By the selection of the smoothness-weight parameter allowing a sufficient number of iterations we can get better result.

\subsection{Block-matching}

Block-matching is employed to find blocks that contain high correlation because its accuracy is significantly impaired by the presence of noise. We utilize a block-similarity measure which performs a coarse initial denoising in local 2D transform domain. In this method image is divided into blocks and noise or blur is removed from each block.

\subsection{LPA-ICI algorithm}

The LPA-ICI algorithm is nonlinear and spatially-adaptive with respect to the smoothness and irregularities of the image and blurs operators. Simulation experiments demonstrate efficiency and good performance of the proposed Deconvolution technique. 


\subsection{Wiener filter}

Wiener filter includes both the degradation function and statistical characteristics of noise into the restoration process. The method is considered on the basis of images and noise as random processes and the objective is to find an estimate function of the uncorrupted image $f$ such that wiener filter minimize the mean square error. It is assumed that the images and the noise are uncorrelated; that one or the other has zero mean and that the gray levels in the degraded image is linear function of estimation.[2]

\subsection{Deconvolutionusing Regularized Filter (DRF)}

Deconvolution by Regularized filtering [2] is another category of Non-Blind Deconvolution technique. When constraints like smoothness are applied on the recovered image and limited information about the noise is known, then regularized Deconvolution is used effectively. The degraded image is actually restored by constrained least square restoration by using a regularized filter. In regularized filtering less prior information is required to apply the restoration. Regularization can be a useful tool, when statistical data is unavailable. Moreover, this framework can be extended to adapt to edges of image, noise that is varying spatially and other challenges.

\subsection{Lucy- Richardson Algorithm Techniques}

Image restoration method is divided into two types one is blind and other is non blind deconvolution. The non-blind deconvolution is one in which the PSF is known. The RichardsonLucy deconvolution algorithm has become popular in the fields of medical imaging and astronomy. Initially it was found in the early 1970's from byes theorem by Lucy and Richardson. In the early 1980's it was redeliver by Verdi as an algorithm to solve emission tomography imaging problems, in which Poisoning statistics are dominant. Lucy Richardson is nonlinear iterative method. During the past two decades, this method have been gaining more acceptance as restoration tool that result in better than those obtained with linear methods.

Thus for restored image of good quality the Number of iterations is determined manually fore very image as per the PSF size.

The Richardson-Lucy algorithm is an iterative procedure for recovering a latent image that has been the blurred by Known PSF.

$$
c_{i}=\sum p_{i j} u_{j}(6)
$$

Where $p_{i j}$ is the point spread function (the fraction of light coming from true location $j$ that is observed at position $i), u_{j}$ is the pixel value at location $\mathrm{j}$ in the latent image, and $c_{i}$ is the observed value at pixel location i. The statistics are performed under the assumption that $u_{j}$ are Poisson distributed, which is appropriate for photon noise in the data. The basic idea is to calculate the most likely $u_{j}$ giventhe observed $c_{i}$ and known $p_{i j}$.It is iterative approach of image restoration. This technique is used where noise function is not known.[4]

\section{Point Spread Function(PSF)}

Point Spread Function (PSF) is the degree to which an optical system blurs (spreads) a point of light. The PSF is the inverse Fourier transform of Optical Transfer Function(OTF) in the frequency domain, the OTF describes the Response of a linear, position-invariant system to animpulse.OTF is the Fourier transfer of the point (PSF). [5]

\subsection{Neural Network Approach}

Neural network is a form of multiprocessor computer system, with simple processing elements, interconnected group of nodes. These

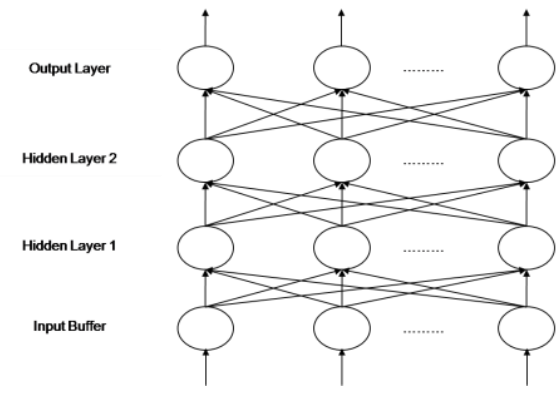

Fig 3: Artificial neural network

Interconnected components are called neurons, which send message to each other. When an element of the neural network fails, it can continue without any problem by their parallel nature. [9]

ANN provides a robust tool for approximating a target function given a set input output example and for the reconstruction function from a class images. Algorithm such as the Back propagation and the Perception use gradient- decent techniques to tune the network parameters to best-fit a training set of inputoutput examples. Back propagation neural network approach for image restoration is capable of learning complex non-linear function this method calculate gradient of function with respect to all weight in function.[10]

\section{Back propagation algorithm}

The BPN contain three layers. Input layer, hidden layer and output layer. During training phase data is given into input layer. In each layer weight is given to each node in a layer. Then weight is summed up and given to next hidden layer.The output of hidden layer act as input for next layer. Once Training phase is completed then it is easy to estimate amount of blur in image.

\section{PERFORMANCE PERAMETER}

\subsection{Peak signal to noise ratio (PSNR)}

PSNR is the ratio between maximum possible power of a signal and the power of distorting noise which affects the quality of its representation. It is defined by:

$$
P S N R=20 \log _{10}\left(\frac{M A X_{f}}{\sqrt{M S E}}\right)(7)
$$

Where $\operatorname{MAX}_{\mathrm{f}}$ maximum signal value that exists is in our original image $\sqrt{M S E}$ is root mean square error.

\subsection{Mean square error (MSE)}

The MSE is the cumulative square error between the encoded and the original image defined by:

$M S E=\frac{1}{m n} \sum_{0}^{m-1} \sum_{0}^{n-1}\|f(i, j)-g(i, j)\|^{2}(8)$

Where, $\mathrm{f}$ is the original image and $\mathrm{g}$ is the uncompressed image. The dimension of the images is $\mathrm{m} x \mathrm{n}$. Thus MSE should be as low as possible for effective compression.

\section{CONCLUSION}

There are many deblurring techniques. After conducting literature survey on various image deblurring techniques proposed by different researchers. Restoration or Deblurring blur from images is a difficult problem to resolve. It is concluded from above techniques that Lucy and wiener filter 
give better results as compare to other techniques. For further advancement we try to apply neural network on these techniques. These Results are concluded on the basis of MSE and PSNR parameters.

\section{REFERENCES}

[1] Er.Jyoti Rani, Er.Sarabjeet Kaur.Volume 4, Issue 1, January 2014.International Journal of Advanced Research in Computer Science and Software Engineering,

[2]Er. Priya Tiwari, Dr. Naveen Dhillon and Er. Kuldeep Sharma.Vol.3, no. 4, 1.July 2013.Analysis of Image Restoration Techniques for Developing Better Restoration Method.

[3] Birwa Raja Mohapatra, Ansuman Mishra, Sarah KumarRout.Vol.2,No.3, March 2014.A Comprehensive Review on Image Restoration Techniques.

[4] Mathri thakur, Shilpa Chadury.Volume 14 Number 4 - Aug 2014.Image Restoration Based On Deconvolution by Richardson Lucy Algorithm. International Journal of Engineering Trends and Technology (IJETT).

[5] R.Pushpavalli1 and G.Sivarajde2.Vol.4, No.2, April 2013.A hybrid filtering technique for eliminating uniform noise and impulse noise in digital image.Signal \& Image Processing. An International Journal (SIPIJ)

[6]. Giacomo Boracchi and Alessandro Foi. Vol. 21, no. 8, August 2012. Modelingthe Performance of Image Restoration from Motion Blur. IEEE Transaction on image processing.
[7] Ryo Nakagaki, Member, IEEE, and Aggelos K. Katsaggelos, Fellow. vol no. 9, September 2003 .A VQ-Based Blind Image Restoration Algorithm, IEEE transaction on image processing.

[8] Dr.P.Subashini Associate Professor. 2221-0741 Vol. 1, No. 6, 277-282, 2011.Image Deblurring Using Back Propagation Neural Network.World of Computer Science and Information Technology Journal (WCSIT) ISSN.

[9]. Neeraj Kumar, Rahul Nallamothu, Amit Sethi.september 2012 Neural Network Based Image Deblurring. neural network application in electrical engineering.

[10]. S.K. Satpathy, S.K. Nayak, K. K. Nagwanshi, S. Panda, C. Ardil. Vol:4 No:1, 2010. An Adaptive Model for Blind Image Restoration using Bayesian Approach. International Journal of Electrical, Robotics, Electronics and Communications Engineering

[11]. S. P. K. Dutta and N. C. Roy, vol. 47, no. 4, 1998.A BlindDeconvolution Approach for Chromatographic and Spectroscopic Peak Restoration. IEEE Transactions on Instrumentation and Measurement

[12]A.M.Raid1, W.M.Khedr2, M.A.El-dosuky1 and Mona Aoud1.Vol. 4, No.3, June 2014. Image restoration based on morphological operations. International Journal of Computer Science, Engineering and Information Technology (IJCSEIT)

[13] Nachtegeal, Waken. karre, E.E.2005. IEEE Explore Fuzzy filters of noise reduction. The case of Gaussian noise. 